\title{
Können Kommunen das Klima retten?
}

\author{
Rezension zu Cindy Sturm (2019): Klimapolitik in der Stadtentwicklung. Zwischen
}

diskursiven Leitvorstellungen und politischer Handlungspraxis. Bielefeld: transcript.

Abb. 1 Titel des Buches (Quelle: transcript)

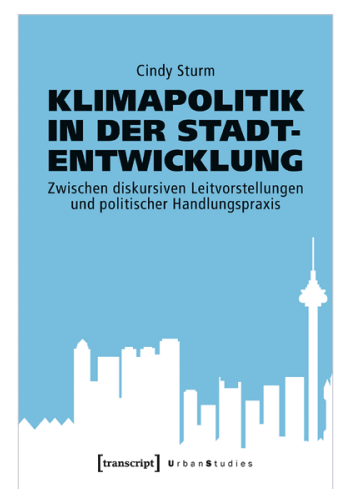

Klimaschutz ist in den wenigen letzten Jahren zu einem dominanten politischen Topos geworden. Insbesondere die Schulstreik-Bewegung der Fridays for Future (FFF) hat die öffentliche Aufmerksamkeit auf die Erkenntnisse der internationalen Klimaforschung gelenkt. Diese hat im Vorfeld der internationalen Klimakonferenzen eingefordert, dass endlich „nie dagewesene Maßnahmen" ergriffen werden müssen, um die für den Fortbestand der menschlichen Zivilisation - so wie wir sie bisher kennen - bedrohlichen Auswirkungen der Erhitzung der Erdatmosphäre auf ein kontrollierbares $\mathrm{Maß}$ zu begrenzen. Die international bestens vernetzten jungen sozialen Bewegungen wie FFF oder Extinction Rebellion haben dabei in letzter Zeit auch die kommunale Ebene als Adressatin für ihre Aktionen entdeckt: Sie fordern von den Kommunen, den Klimanotstand auszurufen, und nutzen Partizipationsmöglichkeiten wie Bürger*innenversammlungen, um dieser Forderung auch durch Beschlüsse Nachdruck zu verleihen. Immerhin haben sich laut Umweltbundesamt mittlerweile mehr als 40 Kommunen per Beschluss selbst verpflichtet, dem Klimaschutz eine höhere Priorität einzuräumen als bisher (Stand 6.2.2020, vgl. Umweltbundesamt 2020). Dabei hat die Ausrufung des ,Klimanotstands“ allenfalls eine symbolische Bedeutung und entfaltet keine rechtlich bindende Wirkung. Vielmehr wird über diese Aktivitäten ein Beitrag zur diskursiven (Re-)Konstruktion eines Politikfeldes auf kommunaler Ebene geleistet und eine Steigerung der Bedeutung des Klimaschutzes auch auf kommunaler Ebene erzeugt.

Für eine wissenschaftliche Einordnung solcher Prozesse bietet das kürzlich erschienene Buch von Cindy Sturm zahlreiche wertvolle Hinweise. Ausgangspunkt ihrer Arbeit ist die Feststellung, dass durch internationale Abkommen den Kommunen schon seit mehreren Jahrzehnten eine bedeutende Rolle bei der Umsetzung umwelt- und klimapolitischer Zielsetzungen zugewiesen wird, diese aber auf lokaler Ebene höchst unterschiedlich aufgegriffen und ausgefüllt wird. Um eine Erklärung für die unterschiedliche Umsetzung klimapolitischer Ziele und Maßnahmen in städtischen Kontexten zu finden, führt Cindy Sturm zwei vergleichende Fallstudien durch: in einer ost- und in einer westdeutschen mittleren Großstadt (Münster und Dresden). Dabei 
interessiert sie insbesondere, welche Diskurse um Klimapolitik sich seit 1997 in der Stadtentwicklungspolitik konstituiert und welche Formen der Steuerung sich etabliert haben, um die formulierten klimapolitischen Ziele in Praktiken der Stadtentwicklungspolitik und Planung zu übersetzen.

Die Tatsache, dass Städten die Aufgabe und die Verantwortung zugeschrieben wird, einen Beitrag zum Klimaschutz zu leisten und gar eine zentrale Umsetzungsebene für die nationalen und internationalen Klimaziele zu sein, ist für Sturm nicht selbstverständlich, sondern Ergebnis von politischen Aushandlungsprozessen und Ausdruck gesellschaftlicher Machtverhältnisse. Um zu verstehen, warum sich vor diesem Hintergrund Klimapolitiken als städtische Politiken konstituieren und wie sie sich in unterschiedlichen Städten unterschiedlich ausprägen, stützt sich Sturm auf einen Mix an theoretischen Konzepten: Die Perspektive der urban policy mobility ermöglicht ihr einen Zugang zu unterschiedlichen lokalen Variationen in der Klimapolitik auf städtischer Ebene. Demzufolge sind Städte keine statische Maßstabsebene politischen Handelns, die vorgegebene Politikkonzepte einer zentralen staatlichen Ebene einfach nur umsetzen. Vielmehr verändern sich politische Modelle und Strategien auf ihrem Weg auf die Ebene der Städte und werden dort in komplexen Kommunikationsprozessen durch spezifische Machtmechanismen und eingeschliffene Alltagspraktiken modifiziert. Übergreifende Politiken werden in verschiedenen Städten folglich unterschiedlich aufgegriffen und selektiv verarbeitet. Somit werden die jeweiligen Kontextbedingungen und Aushandlungsprozesse herausgestrichen und Unterschiede in der lokalen Politikproduktion können erklärt werden.

Die zweiten und dritten konzeptionellen Säulen der Arbeit bilden die Diskurstheorie und die Gouvernementalitätstheorie in Anlehnung an Michel Foucault. Diskurstheoretische Zugänge lenken den Blick darauf, welche Bedeutungen wirkmächtig werden und wie sich diese über die Zeit verändern. Klimapolitiken sind als Ergebnis diskursiver Konstruktionsprozesse zu verstehen, die sich auch verändern könn(t)en. Aus der Perspektive stadtentwicklungspolitischer Diskurse gilt es dann herauszuarbeiten, welche Diskursstränge um Klimapolitik deutungsmächtig werden und wie diese in spezifischen städtischen Konstellationen variieren. Durch die Verbindung einer gouvernementalitätstheoretischen Perspektive mit der Diskurstheorie soll es möglich gemacht werden, die wechselseitige Konstitution von Wissensordnungen und Regierungstechniken in den Mittelpunkt zu rücken. Cindy Sturm interessiert dabei vor allem, welche Rationalitäten und Problematisierungen erzeugt werden, welche Regierungstechnologien der Fremdund Selbstführung sich herausbilden, wie verantwortliche Subjekte hervorgebracht werden und wie mit widerständigen Praktiken verfahren wird?

Dieser komplexe analytische Forschungszugang wird von Cindy Sturm in drei Schritten mit differenzierten methodischen Verfahren umgesetzt. Um übergeordnete sprachliche Muster der Klimapolitik herauszuarbeiten, führt sie ein korpuslinguistisches Analyseverfahren von bundespolitischen und stadtpolitischen Dokumenten durch (Makroperspektive). Die zweite Perspektive (Mikroperspektive) wird durch Interviews mit politischen Entscheidungsträger*innen und Verwaltungsmitarbeiter*innen sowie durch Auswertung städtischer Berichte in den beiden untersuchten Städten erzeugt. Mithilfe von qualitativem Kodieren mit MaxQDA verschafft sich die 
Autorin einen Überblick über artikulierte Themen, Problematisierungen und Praktiken in den beiden Fallstädten Münster und Dresden. Mit einer Aussagenanalyse erfasst sie heterogene Stimmen, Konflikte und Widerstände.

Dabei gelingt es ihr zunächst spezifische Konjunkturen von Begriffen in der umwelt- und klimapolitischen Diskussion herauszuarbeiten, die den lokalen Diskurs rahmen. Es fällt auf, dass insbesondere in Dresden wie in ganz Ostdeutschland der Stadtumbau über Jahre hinweg umwelt- und klimapolitische Themen in den Hintergrund gedrängt hat. Dies hat in Dresden schließlich auch zu einer spezifischen Form des Umgangs mit Klimapolitiken geführt, die eher auf einer fachlichen Verwaltungsebene im Hintergrund aufgebaut und umgesetzt worden sind und weniger nach außen getragen wurden. Zudem gibt es in Dresden durchaus mächtige Gegenbewegungen, da die lokalen Vertreter*innen der bürgerlichen Parteien sich von ihrer bundespolitischen Linie deutlich abwenden und skeptischen Positionen gegenüber einem menschengemachten Klimawandel viel Raum bieten. Erst mit dem jüngsten Regierungswechsel in der Stadtpolitik hin zu den Parteien aus dem Spektrum der linken Mitte wurde auch ein Diskurswechsel verbunden und die Notwendigkeit eigenständiger Klimapolitiken stärker betont.

In Münster hingegen ist der klimapolitische Diskurs seit langem und kontinuierlicher präsent und wird in Kongruenz mit neoliberalen Wettbewerbsstrategien sogar als Imagefaktor aktiv in der Kommunikationspolitik und Außendarstellung der Stadt hervorgehoben. Münster wird vom Bund aktiv in eine Strategie der Verantwortungsabgabe auf die Ebene der Städte einbezogen und in modellhafte Projekte eingebunden, die herausragende Praxisbeispiele für Klimapolitiken auf städtischer Ebene produzieren sollen. Die langjährige Vorbildrolle führt in Münster dazu, dass auch Vertreter*innen bürgerlicher Parteien den Klimaschutz als wichtige Aufgabe hervorheben.

In beiden Städten wurden zudem wesentliche Impulse in den lokalen Debatten durch Vereinbarungen auf internationaler Ebene - wie die Globale Nachhaltigkeitsagenda der UN mit ihren 17 Sustainable Development Goals (Agenda 2030) oder dem UN Klimaschutzabkommen von Paris aus dem Jahr 2015 - ausgelöst. Solche Dokumente und ihre Umsetzung auf nationalstaatlicher Ebene weisen den Kommunen Verantwortlichkeiten zu, einen wesentlichen Beitrag zur Umsetzung der Ziele zu leisten.

Cindy Sturm zeigt in ihrer Arbeit auf, dass diese Impulse in beiden Städten jedoch unterschiedlich ,ankommen' werden und zu unterschiedlichen Ausformungen konkreter Klimapolitik führen. Während in Dresden Umweltund Klimapolitiken stärker mit Städtebauförderprogrammen und Stadtumbaumaßnahmen verbunden sind und Regierungstechnologien der agency im Sinne von empowerment und Steigerung der Handlungsfähigkeit repräsentieren, sind es in Münster Regierungstechnologien der Performanz, die dominieren. Diese vermitteln sich über die Teilnahme an Zertifizierungsverfahren, Benchmarks, Wettbewerben, Modellprojekten wie die 100 Prozent Modellkommune (100 Prozent Energieversorgung aus erneuerbaren Energiequellen) und Best-Practice-Verfahren. Dies verbindet sich mit der Herausbildung spezifischer Kompetenzen und Strategien der entscheidenden Subjekte in den Verwaltungen und in der Politik. In Münster kann die zugeschriebene Musterrolle in der Klimapolitik als individueller Erfolg der Akteure herausgearbeitet und expressiv zu Marketingzwecken 
eingesetzt werden. In Dresden müssen die Akteure, die erfolgreich Klimaschutzpolitik betreiben wollen, diese eher in leichter vermittelbaren komplexen Projekten wie Stadtumbauprojekte ,einbauen " und dort ,verstecken'.

Auch spielen in beiden Städten unterschiedliche lokal spezifische Erfahrungen mit extremen Wetterereignissen eine große Rolle in der Ausformung der jeweiligen städtischen Diskurse zur Klimapolitik. Insbesondere die katastrophalen Hochwasser in Dresden haben Gelegenheitsfenster für lokale Klimapolitiken aufgestoßen und die Klimapolitik stärker in den Vordergrund des stadtpolitischen Diskurses gerückt. In Münster haben die deutlich schwächeren aber überraschenden und ungewohnten Hochwasserereignisse eher als Bekräftigung des eingeschlagenen Weges fungiert.

Sturm gelingt es in ihrer Arbeit in methodisch überzeugender Weise einen sozialwissenschaftlich fundierten Einblick in das Politikfeld des kommunalen Klimaschutzes zu generieren. Dabei deckt sie neoliberale Strategien der Verantwortungsdelegation (vom Bund auf die Städte, von der Politik auf die Zivilgesellschaft) auf. Darüber hinaus gelingt es ihr, unterschiedliche Muster städtischer Klimapolitiken herauszuarbeiten und zu begründen. Die konzeptionelle Kombination aus den Theorien der urban policy mobility, der Diskurstheorie und der Gouvernementalitätstheorie eröffnet ein vielversprechendes Forschungsprogramm zur Erklärung divergenter Muster lokaler Politiken und zur fundierten Analyse spezifischer lokaler Ausprägungen. Diese Monographie zeigt, dass strukturelle Aspekte (vorherrschende Diskurse und Konkurrenzen um Deutungshoheiten) einerseits mit subjektbezogenen Perspektiven (Subjektformierungen) und Herrschaftstechnologien (vorherrschende Regierungstechnologien) andererseits gewinnbringend verbunden werden können. Dies erlaubt Einblicke in die Konstitution des Politikfelds städtischer Klimaschutz, den sich auch soziale Bewegungen für ihre Strategiekonstruktion nutzbar machen können. So wird deutlich, dass einerseits die jeweilige Formierung der städtischen Klimapolitik vor dem Hintergrund der lokal spezifisch ausgeformten Diskurse verstanden werden muss, um geeignete Anknüpfungspunkte für wirksame zivilgesellschaftliche Bewegungen zu finden. Andererseits zeigen die Untersuchungen von Cindy Sturm, dass die Gestaltung der lokalen Diskurse offen ist gegenüber Einflüssen von außen. Zivilgesellschaftlicher Druck könnte an geeigneter Stelle zu einem Katalysator und Accelerator für städtische Klimapolitiken werden und zur Öffnung von Gelegenheitsfenstern beitragen. Momentan versuchen zahlreiche Initiativen, mit der Forderung nach Ausrufung eines Klimanotstands, den lokalen Diskurs zu beeinflussen und ambitionierteres Handeln einzufordern. Es wird sich zeigen, wie sich diese Forderungen, die überall nach sehr ähnlichem Muster erhoben werden, in den jeweiligen stadtpolitischen Kontexten modifizieren und zu mobilen urbanen Politiken werden.

\section{Autor_innen}

Manfred Miosga forscht zur Frage, welcher Beitrag zur Nachhaltigkeitstransformation auf der Ebene der Stadt- und Regionalentwicklung geleistet werden kann.

manfred.miosga@uni-bayreuth.de 


\section{Literatur}

Cindy Sturm (2019): Klimapolitik in der Stadtentwicklung. Zwischen diskursiven Leitvorstellungen und politischer Handlungspraxis. Bielefeld: transcript.

Umweltbundesamt (2020): Deutsche Kommunen rufen den Klimanotstand aus. https:// www.umweltbundesamt.de/themen/klima-energie/klimafolgen-anpassung/anpassungan-den-klimawandel/anpassung-auf-kommunaler-ebene/deutsche-kommunen-rufenden-klimanotstand-aus\# (letzter Zugriff am 17.02.2020). 